\title{
Lenguaje y comunicación de insectos en Rhapsody in Stephen's Green de Myles na gCopaleen
}

Fecha de recepción: 26/5/2020. Fecha de aceptación: 7/4/2021.

\section{Resumen}

Rhapsody in Stephen's Green fue escrita por Myles na gCopaleen, también conocido como Flann O’Brien o Brian O’Nolan. Este texto dramático es una adaptación de la obra de los hermanos Čapek: života hmyzu (1921) y, aunque le debe la estructura episódica y el concepto general a su fuente checa, se caracteriza por su uso del lenguaje y su relación con el regionalismo irlandés. Específicamente, la relación de los insectos con su espacialidad y en cómo la oralidad proporciona un tipo específico de identidad como en el caso de los escarabajos de Dublín y los grillos de Cork, entre otros.

La producción literaria de Myles na gCopaleen es conocida por cuestionar el significado y el uso del lenguaje humano y, en particular, en Rhapsody in Stephen's Green explora la posibilidad de un lenguaje animal y su relación con la sociedad irlandesa. Si el único límite entre el hombre y el animal podría ser trazado por el lenguaje y la capacidad nominal de la palabra, ¿cómo es que el uso particular del lenguaje por parte de estos insectos cuestiona el significado y el desempeño de las interacciones orales junto con su correspondencia identitaria? 
Insect language and communication in Myles na gCopaleen's Rhapsody in Stephen's Green

\begin{abstract}
Rhapsody in Stephen's Green was written by Myles na gCopaleen, also known as Flann O'Brien or Brian O'Nolan. This dramatic text is an adaptation of the Čapek brothers' play: života hmyzu (1921) and, although it owes its episodic structure and general concept to its Czech source, it is characterized by its use of language and its relationship to Irish regionalism. Specifically, the relationship between insects and their spatiality, and the way their orality provides a specific form of identity, as in the case of the Dublin beetles and the Cork crickets, among others.

The literary production of Myles na gCopaleen questions the significance and the use of human language and, in particular, in Rhapsody in Stephen's Green it explores the possibility of an animal language and its relationship to Irish society. If the only boundary between man and animal could be established by language and the nominal capacity of the word, how does these insects' particular use of language challenge the meaning and performance of oral interactions along with their identity correspondence?
\end{abstract}

Keywords: Rhapsody in Stephen's Green; Myles na gCopaleen; language; identity; insects

\title{
I. Introducción
}

En julio de 1942, Hilton Edwards le escribe a Brian O’Nolan para proponerle hacer una nueva traducción de The Insect Play: "What about an Irish version with a tramp speaking as an Irishman would and with various insects speaking as Irish insects and not as cockneys?" (Long, 2018, p. 122). Mediante este intercambio epistolar el productor deja en claro que el texto original necesita de una adaptación a la escena irlandesa y que sería un medio perfecto para el autor debido a que los personajes se expresan a través de diálogos. Qué mejor que los retratos cliché de la sociedad irlandesa de Myles, una de las tantas caras ficcionales de o’Nolan, para dar voz a los insectos de la obra de los hermanos Čapek.

Rhapsody in Stephen's Green (The Insect Play) pone en escena los sucesos que ocurren en Stephen's Green, parque del centro de la ciudad de Dublín, luego del cierre de sus puertas por la noche. En el prólogo, se plantea un espacio que se deshace de toda su población humana para dar paso a las escenas protagonizadas por los insectos que habitan el parque durante los tres actos de la obra. Tal como en el caso de su fuente, Rhapsody in Stephen's Green establece una continuidad entre las diferentes partes del texto dramático a partir del personaje del vagabundo que presenta un estado de borrachera lindero al sueño. ${ }^{1}$ Cada acto 
Lenguaje y comunicación de insectos en Rhapsody...

se va a centrar en grupos diferentes de insectos y en las situaciones particulares en las que están envueltos cada uno de estos. Por ejemplo, el par de abejas del primer acto que desea picar algo para poder morirse, la pareja de escarabajos del segundo acto que pierde sus ahorros o el conflicto bélico entre diferentes tipos de hormigas en el último acto.

El uso del lenguaje en los textos de Brian O’Nolan, a través de sus figuras autorales ficcionales, "questions the very definitions of meaning and human language” (Coulouma, 2015, p. 10) y en Rhapsody in Stephen's Green encuentra en la adaptación del texto un medio perfecto para confeccionar un uso recargado de muletillas propias de la oralidad que a su vez emulan distintos regionalismos irlandeses que son correspondidos con los distintos tipos de insectos. De esta manera, si aquellos que son dotados de esta capacidad lingüística y comunicativa son los insectos, surge una pregunta fundamental sobre el estatuto del lenguaje utilizado. Ahora bien, el posible límite que constituye la separación entre la esfera humana y la animal es producto de reflexión y comentario desde Aristóteles hasta la actualidad. ${ }^{2}$ El lenguaje parece ser un punto decisivo para esta demarcación: "lo que discrimina al hombre del animal es el lenguaje, pero este no es un dato natural innato en la estructura psicofísica del hombre, sino una producción histórica que, como tal, no puede ser propiamente asignada al animal ni al hombre" (Agamben, 2006, p. 73). Por lo tanto, si un posible límite entre el hombre y el animal puede ser trazado por el lenguaje y la capacidad nominal de la palabra, el uso particular del lenguaje por parte de los insectos de Rhapsody in Stephen's Green cuestiona el significado y el desempeño de las comunicaciones lingüísticas junto con su correspondencia identitaria nacional, es decir, irlandesa. ${ }^{3}$

\section{La figura ficcional de Myles na gCopaleen}

El 25 de enero de 1943, en el estreno de Faustus Kelly, ${ }^{4}$ el público pide en el saludo final por el autor, pero se ve rápidamente sorprendido al ver delante de sus ojos a una figura caricaturesca y de apariencia similar a la de una escenificación de hombre irlandés tradicional: un hombre con pipa, caubeen -boina típica nacional-y chaqué. Esta figura era un actor del Abbey Theatre quien hizo un pequeño gesto a la audiencia y se esfumó. La ficción planteada por Brian O'Nolan extendía los límites de la representación hasta su propia figura.

onírico del vagabundo, esta no será tenida en cuenta para el presente análisis. Esto se debe a que, independientemente de si los insectos son parte de la psiquis del vagabundo o reales para el contexto dramático, no afecta el trabajo sobre la relación de categorías hombre-insecto en sus diferentes posibilidades, su uso del lenguaje y su correspondencia identitaria.

2 Entre estos se encuentran Aristóteles (2000); Heidegger (2007); Nietzsche (1998); Derrida (2008); Agamben (2006) y Cragnolini (2016), entre tantos otros.

3 A pesar de hacer mención a la primera representación de la obra, el presente trabajo busca hacer una lectura crítica del texto dramático Rhapsody in Stephen's Green (The Insect Play).

4 Faustus Kelly es la segunda obra de Myles na gCopaleen. Esta es una obra de tres actos que hace una parodia cómica del texto tradicional de Christopher Marlowe, pero que está inmersa en los quehaceres de una figura de la política irlandesa y su deseo de avanzar en aquel ámbito. 
Esta noción caricaturesca y ficticia del autor ya estaba presente en el seudónimo utilizado para firmar su producción dramática. ${ }^{5}$ Todas las obras de teatro de Brian O'Nolan de los años 40 fueron escritas, publicadas y producidas bajo el nombre Myles na gCopaleen, así como también las entregas del Irish Times de Cruiskeen Lawn. El nombre Myles na gCopaleen, tal como representa la figura ficticia que aparece en el teatro, refiere, por un lado, a un personaje de la literatura tradicional irlandesa y, por otro lado, a la naturaleza descriptiva del nombre en gaélico irlandés, personificando el estereotipo de hombre rural. Explica Tracy en su introducción a Rhapsody in Stephen's Green (The Insect Play) que

Myles na gCopaleen, Myles of the Little Horses, or, as he himself was to insist, Myles of the Ponies, was born in 1829, as the resourceful and loquacious horsetrader in Gerald Griffin's melodramatic novel The Collegians. He was born again three times: as the leading character in Dion Boucicault's play The Colleen Bawn (1860), freely adapted from Griffin's novel; as a tenor role in Sir Julius Benedict's opera The Lily of Killarney (1862), adapted from Griffin and Boucicault; and finally, yet again, in October 1940, when Brian O'Nolan borrowed his identity to write a column for The Irish Times (1994, p. 6).

Cruiskeen Lawn, publicada desde octubre de 1940 tenía como autor también a Myles na gCopaleen, una figura que ganó popularidad en Dublín junto con su mirada satírica y los personajes presentados en su columna. No obstante, Myles era uno de los tantos nombres utilizados por Brian O'Nolan para firmar sus textos. ${ }^{6} \mathrm{El}$ uso de seudónimos presenta una nueva figura de autor que desmonta un posible estatuto de singularidad creadora y única, proponiendo a su vez una posible tradición colectiva y minoritaria; tradición anclada en un uso menor de la lengua que deja en evidencia el fracaso, con la conformación de la república, de las políticas lingüísticas establecidas en favor del renacimiento irlandés. ${ }^{7}$ Así, tanto el uso del nombre Myles na gCopaleen y su significado en relación con Rhapsody in Stephen's Green, forman parte de un proyecto de obra más amplio por parte de O’Nolan.

Myles na gCopaleen, aparte de su publicación regular en el Irish Times, escenifica entre los años 1942 y 1943 tres obras teatrales escritas por él, Faustus Kelly, Thirst y Rhapsody in Stephen's Green (The Insect Play), que no se consideran muy exitosas. ${ }^{8}$ Carol Taaffe sostiene que "at the time both [Faustus Kelly and The Insect Play]

5 Vale aclarar que esta distorsión de la identidad autoral que presenta el uso de seudónimos estaba presente no solo en la producción dramática de Brian O'Nolan, sino en el resto de su obra. A saber, su primera novela At Swim-Two-Birds está firmada bajo el nombre Flann O’Brien, uno de los seudónimos más utilizados por el autor.

6 Maebh Long, para ilustrar esta cuestión, se pregunta: “Is he Brian O’Nolan, Brian Ó Nualláin, Myles na gCopaleen, Myles na Gopaleen, Lord Nolan of Santry, Flann O’Brien, George Knowall, John James Doe, An Broc, Brother Barnabas, Count O'Blather, or any of the other names he harassed his own alter egos under in The Irish Times?" (2014, p. 108)

7 Resulta imperante no perder de vista la categoría de literatura menor, presentada por Gilles Deleuze y Félix Guattari en Kafka por una literatura menor. La literatura menor es una "literatura que una minoría hace dentro de una lengua mayor" (1990, p. 28). Los casos irlandeses que aparece mencionados en el texto son el de Joyce y su uso del inglés, y el de Beckett y su uso del francés.

8 La crítica basa este juicio en que cada obra cuenta con muy pocas representaciones en las producciones originales mencionadas. Jernigan (2013) menciona que Faustus Kelly fue representada en el Abbey Theatre por casi dos meses a partir del 25 de enero de 1943 y que Thirst fue encargada como entretenimiento navideño dentro de la programación del Gate Theatre en 1942 y, por lo tanto, contó con una sola función en esa ocasión. Rhapsody in Stephen's Green, por su parte, fue estrenada en el Gaiety Theatre y representada del 22 de marzo a 27 de marzo de 1943. 
Lenguaje y comunicación de insectos en Rhapsody...

were commercial failures, and what might have initially seemed a promising vein of work proved to be little more than a short division in his career" (2008, p. 175). De manera sorpresiva, la producción contemporánea de Flann O’Brien, seudónimo utilizado principalmente para publicación de novelas, parece haber sido más fructífera para las representaciones teatrales debido a que fue posteriormente adaptada a escena en numerosas oportunidades. ${ }^{9}$

Así como la figura de Myles fue polémica en el estreno de Faustus Kelly, Rhapsody in Stephen's Green también tuvo su propia controversia posterior al estreno. Los críticos tenían opiniones encontradas sobre la obra, generalmente duras, calificando su lenguaje como "pointless burlesque in Irish dialect over-emphasised to the point of grotesque exaggeration" (Long, 2017, p. 43). Myles y Gabriel Fallon, crítico teatral de la época, mantuvieron un intercambio en el periódico The Standard sobre el estreno de la obra en cuestión debido a que el autor decide responder ante las acusaciones de obscenidad, de lenguaje indecente y de burlas profanas que se suceden en la obra. La respuesta de Myles a Fallon dice:

Since your paper honours Mr. Fallon with the role of critic, I think he is entitled to denounce every single play he sees if he feel that way about it, however much his disapproval may be the result of ignorance or mental immaturity (Tracy, 1994, p. 17).

No obstante, para enfatizar el juego ficticio y polémico alrededor de la producción, el mismo periódico publica una carta de un miembro de la audiencia que se cree que es otra de las tantas máscaras del autor criticando eso que Myles defiende en las mismas hojas. ${ }^{10}$ La propia mirada y discurso público es, nuevamente, consecuencia de una creación ficticia de O’Nolan. De forma más notable, la polémica en torno a la recepción de la primera representación y, en particular, en relación al uso del lenguaje es de suma importancia para enmarcar el análisis de la naturaleza discursiva de las interacciones de los insectos en el texto dramático.

\section{El espacio irlandés en Rhapsody in Stephen's Green}

Rhapsody in Stephen's Green (The Insect Play) suele ser descripta como una adaptación libre de života hmyzu (1921) de Karel Čapek y Josef Čapek. Según Robert Tracy (2012), Myles les debe a los hermanos Čapek la estructura episódica y el concepto general de la obra. ${ }^{11}$ La fuente original checa, estrenada por primera

9 Entre ellas se pueden mencionar las producciones de At Swim-Two-Birds del Abbey Theatre de los años 1971 y 1998, la producción de 1994 de la compañía teatral inglesa Ridiculusmus y la versión representada por la Blue Raincoat Theatre Company en Sligo en 2009

10 Indudablemente, el uso de diferentes nombres por parte de O'Nolan para publicar notas de opinión sobre diferentes temas en medios gráficos fue aquello que llamó la atención de R. M. Smyllie, el editor de The Irish Times, que decidió contratarlo como columnista de la mencionada Cruiskeen Lawn. 
vez en Brno el 3 de febrero de 1922, cuenta con un vagabundo que se duerme en el bosque y sueña con una serie de insectos humanizados que comentan sobre la vida de posguerra en Checoslovaquia. Si bien en el prólogo y en el acto I Myles se separa fuertemente de la obra fuente, los actos II y III parecen seguir un poco más de cerca el texto original. Los cambios suelen darse en qué tipo de insectos se presentan y en cómo estos interactúan con el vagabundo y su alrededor. ${ }^{12}$ Asimismo, una de las principales variaciones sobre su fuente refiere al uso del lenguaje y su relación con el regionalismo irlandés. Específicamente, la relación de cada especie de insecto con una ciudad de Irlanda y la forma en que su oralidad retrata una forma específica de identidad.

En este sentido, desde la alusión del título a Stephen's Green hay una referencia directa de la geografía local y cercana al espectador de la primera representación en el Gaiety Theatre. Es decir, un deseo de anclar el texto dramático, y sus posibles representaciones, a un espacio irlandés. Esta intención de acercar la representación dramática al ámbito local se vislumbra, a su vez, en el propio texto donde "the new context is forcibly presented in the prologue, with the tramp wistfully contemplating the social welfare proposals that were being debated in the Dublin press" (Taaffe, 2008, p. 180). Estas menciones, se concentran, por parte del vagabundo, en las propuestas de "family allowances...and plenty of free insurance" (O’Brien, 2013, p. 166). En particular, esta cita refiere a los debates sobre subsidios sociales que ocurrieron de forma contemporánea a la escritura y primera representación de la obra que se enmarcan en el período que se conoce como The Emergency. ${ }^{13}$ Es así que a la localización geográfica explícita se le suma un entorno político y social contemporáneo al contexto de escritura y de la primera representación.

De esta manera, todos los insectos que se presentan en Rhapsody in Stephen's Green tienen un elemento en común; abejas, escarabajos, grillos y hormigas reproducen conductas humanas desde la comunicación lingüística hasta su apariencia. Es decir, no solo se comunican entre sí utilizando lenguaje humano, entendible y asimilable por los lectores y las posibles audiencias, sino que las didascalias del texto acercan aún más a estos seres a la conducta humana al dar directivas tales como: "the females are distinguished by high-heeled shoes, coloured handkerchiefs round the head” (ibíd., p. 168). En una primera instancia, entonces, hay una clara humanización de los insectos en la acción dramática: son representados de forma antropologizada por actores, se comunican a partir del lenguaje y la apariencia responde a ciertos criterios humanos, como las distinciones de género a partir de usos accesorios específicos.

Como se mencionó anteriormente, los insectos de la obra se relacionan íntimamente con la escena local y esto también se puede ver en cómo las didascalias y

de obras de un acto.

12 Para una estudio más detallado sobre el uso de la fuente, véase Tracy, R. (1994).

13 Es decir, el estado de emergencia en el que vivía Irlanda a pesar de haberse pronunciado como neutral en la Segunda Guerra Mundial. Véase Asensio Peral (2018) y, de forma más general, Girvin (2006) y Hachey (2002), entre otros. 
Lenguaje y comunicación de insectos en Rhapsody...

las grafías del texto les asignan acentos particulares de distintas regiones dentro de Dublín y del resto de Irlanda. Es decir, los insectos se vuelven rápidamente irlandeses y, luego, dublineses o pertenecientes a otras ciudades como Cork o Belfast. Este gesto indudablemente refiere a la escritura de la oralidad que Myles practica en su columna Cruiskeen Lawn, publicada en el diario The Irish Times. Este gesto de plasmar las particularidades de la oralidad revela un interés por destacar un uso disímil que emerge, fonética y rítmicamente, del irlandés y establece un uso menor dentro de una lengua mayor. En palabras de Coulouma:

Most of O'Brien's characters are phonetically at odds with the language they speak. Their dialect, accent and register of speech betray their ethnic, geographic and social inferiority to the colonial norm, revealing a radical inadequacy: their organs of speech are not meant for Standard English (2015, p. 134).

Es así que Rhapsody in Stephen's Green no solo presenta un escenario local, sino que retrata particularidades sociales ligadas a la conformación de identidades específicas dentro de la propia Irlanda. Si se piensa al lenguaje aparejado a ciertas acciones constituidas socialmente, la adquisición de este por parte de los insectos en el texto dramático se encuentra arraigada, entonces, a una correspondencia geográfica y cultural.

\section{Comunicación animal y humana}

Émile Benveniste en Problemas de lingüística general I piensa las posibilidades comunicacionales del lenguaje animal. Al analizar la comunicación entre abejas, ${ }^{14}$ menciona que esta compete "un simbolismo verdadero, si bien rudimentario, por el cual datos objetivos son traspuestos a gestos formalizados, que incluyen elementos variables y de 'significación constante" (1997, p. 60). En otras palabras, los animales establecen una comunicación entre sí aunque esta forma de interacción no podría ser referida de igual manera que el lenguaje que utilizan los humanos. Esto se debe a que los distintos lenguajes utilizados por los hombres establecen una comunicación lingüística. A diferencia de las interacciones animales, los lenguajes humanos no corresponden a situaciones fijas, como podría ser el caso de la danza de las abejas, sino que emplean símbolos y esto implica una capacidad de interpretación ya que no existe una relación natural con aquello que simbolizan.

En el caso de Rhapsody in Stephen's Green, el diálogo, forma esencial del lenguaje humano para Benveniste, es recurrente entre insectos y hombres. Estos insectos hacen uso de esta posibilidad comunicativa humana ya que son capaces de armar y significar discurso según reglas lingüísticas compartidas y, por tanto, al mismo momento con el lector del texto dramático como con la posible audiencia

14 Benveniste toma el estudio que realiza Karl Von Frisch sobre la forma de comunicación que se puede leer en la danza de las abejas. 
en caso de una representación. Es decir, los insectos están inmersos en la lógica del lenguaje porque pueden establecer intercambios comunicativos de tipo lingüístico con otros.

De esta manera, si una de las diferencias constitutivas entre el hombre y el animal podría pensarse en la capacidad del lenguaje desde la lectura de Agamben, en Rhapsody in Stephen's Green puede verse la desaparición de esta misma en los diferentes tipos de intercambio que suceden en la obra. Es así que hay comunicación entre insectos del mismo tipo -el par de abejas Cyril y Cecyl en el acto I-, insectos de diferente tipo -la mujer escarabajo y la mujer grillo en el acto II- e insectos y humanos -las diferentes interacciones que establece el vagabundo con los distintos insectos a lo largo de los actos-.

El vagabundo, entonces, es la figura que se ocupa de este dialogo entre especies y establece este nexo concreto entre lo humano y lo animal. El personaje cuenta con el nombre "tramp" y en ningún momento se lo refiere a partir de un nombre propio. Es decir, aquello que el personaje es lo nombra y lo caracteriza. El vagabundo es el único personaje que aparece tanto en el prólogo y epílogo como en los tres actos de la obra: escapa el control del guarda del parque y sobrepasa el límite de este espacio humano que en su horario de cierre se vuelve un espacio animal.

Al no tener nombre, el vagabundo es una suerte de no-persona, o en el caso de la configuración de la ciudad moderna y estado-nación un no-ciudadano, al margen de su propio ámbito social que actúa como figura fronteriza entre lo humano y lo animal, y tiene sentido que este acceda, a su vez, a aquello que también habita el espacio del parque en sus márgenes. La falta de nombre propio no resulta extraña en la producción de Myles ya que en An Beal Bocht todos los niños de Corca Dhorcha, Gaeltacht ficcional donde transcurre la novela, son bautizados como “Jams O’Donnel” en el colegio. Frente a esto, Long destaca que "there is never an attempt to give the Irish speakers a proper name - they are associated always with superficial descriptors and categories" (2014, p. 108). Y, de esta manera, la ausencia de identidad nominal de los niños en las áreas de habla irlandesa ante la escolarización inglesa hace que sus nombres, los nombres irlandeses, se encuentren por fuera de la ley. De forma similar, la falta de nombre y el lugar marginalizado pueden extenderse en este caso a la figura de vagabundo quien no tiene identidad constituida y habita un espacio deshabitado por los hombres.

Asimismo, la intervención de este personaje en las conversaciones con los insectos organiza y direcciona, sobre todo en el acto II, la acción del texto dramático ya que el vagabundo observa conversaciones de cierto grupo de insectos, interactúa con ellos y luego cambia de grupo de insectos con los que establece nuevamente un dialogo y así con el correr de las escenas. De por sí, este recurso no solo habilita una transición entre los acontecimientos que le ocurren a distintos insectos, sino que habilita la observación y el comentario de la voz humana sobre aquello que está observando en la interacción animal: 
Lenguaje y comunicación de insectos en Rhapsody...

It's very haird... but it's very interesstin'- Them little buggers with all the legs on them is queer men. Don't give a damn for one another - every man for himself. You are me or I'll ate you (O'Brien, 2013, p. 211).

Las palabras del vagabundo revelan una cierta igualdad entre la actividad de los insectos y la de los hombres. Por un lado, se menciona que los insectos son, efectivamente, "hombres raros" y esta visión se refuerza al mencionar que "cada hombre por su cuenta”, aún refiriéndose a los insectos. Por otro lado, esta observación abre con la idea de la dificultad de comprensión sobre la interacción de estas especies -"es muy difícil"- que, asimismo, cierra con esta idea que, ante la dificultad de igualarse con el otro, la única solución es comerse entre sí. De este modo, se manifiesta en estas palabras del vagabundo el hecho que estas relaciones entre animales se establecen desde el punto de vista de un entendimiento social presente en la forma en que el vagabundo entiende distintas interacciones de la esfera animal en relación con la humana.

La reflexión del vagabundo continúa y su discurso permite establecer ciertos parámetros para reflexionar sobre las categorías entre especies mencionadas previamente:

\begin{abstract}
Begob I think I've put me finger on it here. That's the difference between meself and me likes and them lads. We have a system, a proper way of workin'. We have what they call a plan. Every man with his own job, all workin' away together for the good of all. What they call the Nation [...] Human beins' is civilized because they do be workin' for one another and workin' together. But these mad whores here do be atin' one another. And that's just the difference between the two (ibíd., pp. 211-212).
\end{abstract}

Acá, nuevamente, el vagabundo reflexiona entre las diferencias y similitudes que encuentra entre las interacciones de los insectos y las del ámbito humano. Hace énfasis, sobre todo, en la diferencia que ve entre los insectos que observó y con los que dialogó, y en las interacciones propias de los humanos. Según este personaje, los humanos tienen "un sistema" referido a la especialización de la cadena productiva que los diferencia de los insectos -"Every man with his own job, all workin' away together for the good of all"-. ${ }^{15} \mathrm{Y}$, si bien los insectos ocupan parte de un ecosistema, estos no son "civilizados" y no conforman "lo que se llama una nación”, es decir, no comprenden una organización mayor en términos sociales y políticos como enuncia en este caso el vagabundo. Es de notar que el lugar de enunciación del vagabundo, como se mencionó anteriormente, es desde el margen de instituciones humanas, como el mercado y la sociedad, y que la defensa por estas categorías humanas se reviste de un tinte irónico ya que él no parece pertenecer al "good of all" que menciona.

Esta desorganización que menciona el vagabundo en los insectos se ve aún más acrecentada con, nuevamente, el tema de aquello que se come y el énfasis en la

15 Frente a esto, resuena una idea que circulaba ampliamente por aquellos años debido a su novedad y es, por supuesto, la línea de ensamblaje de Ford y la nueva aproximación que esta trae a la producción y a la manera de concebir el trabajo dentro del capitalismo. 
capacidad bárbara que reviste la idea de "comerse el uno al otro". Una vez más, la inflexión entre especies se encuentra en esta idea de no comerse y matarse entre sí, un entendimiento que establece el vagabundo desde su posición enunciativa. No obstante, la posición del vagabundo resulta compleja, ya que él mismo mata a un insecto y habita una sociedad que debate constantemente diferentes propuestas y acciones bélicas en los medios. ${ }^{16}$ Nuevamente, aquí el vagabundo aparece como un personaje de transición que, en su carácter bifronte, se convierte en una no-persona: al encontrarse situado en los márgenes de dos mundos está en la práctica fuera de ellos.

Si bien el vagabundo realiza juicios sobre los insectos que lo rodean, este personaje también es directamente interpelado por la esfera animal: "Do oo eat people up or bite?” (ibíd., p. 201). Una vez más la idea de comer sería una forma de establecer un vínculo entre especies que al parecer no podría establecerse de otra manera. Es más, en el dialogo citado no se reconoce al vagabundo como hombre y se le pregunta por su capacidad de comer o lastimar a otras especies o clases de insectos. Incluso, en este carácter de no-persona o no-ciudadano que habita el vagabundo, el vagabundo cuenta con una descripción sobre su habla: "his accent is a richer Dublin job tan the KEEPER" (ibíd., p. 164). De esta manera, el vagabundo tiene una conformación de identificación espacial, social y cultural similar a los insectos y no una de forma diferente por su calidad de hombre.

Es así que el umbral de la división entre hombre y animal debe revisitarse. El primer criterio de separación establecido en el lenguaje se borra, puesto que el texto dramático presenta insectos humanizados que establecen diálogos entre sí. ¿Estamos, entonces, frente a personajes hombre-animal o animal-hombre? Agamben vislumbra estas posibles combinaciones entre lo animal y lo humano, y argumenta que "el hombre-animal y el animal-hombre son las dos caras de una misma fractura, que no puede ser colmada por una parte ni por la otra" (2006, p. 74).

En cuanto a los intercambios entre ellos a lo largo del texto dramático, los insectos se interpelan entre sí de la manera en la que lo hacen los hombres, es decir, usando formas referenciales como "man" o "people". Aquí, entonces, se realiza una igualdad referencial en el discurso a través de las especies. De este modo, ¿dónde se traza el límite de la comparación entre especies y qué dimensión simbólica conlleva cada una? Según el estudio de Tracy que acompaña la publicación de la obra en 1994, no hay dudas: "though the Capeks left it to the director to decide whether the characters would be people acting like insects, or insects acting like people, Myles is more decisive [...] His humans are insects" (1994, p. 17). Si bien el comentario de Tracy refiere al modo de escenificación de la producción, la decisión en el texto dramático que los actores sean los insectos, y no a la inversa, da cuenta de un gesto que viene desde la escritura; es decir, una

16 Resulta imperante volver a recordar la coyuntura histórica y el estado de emergencia que experimentaba Irlanda en relación a la Segunda Guerra Mundial al momento de escritura del texto. 
Lenguaje y comunicación de insectos en Rhapsody...

intención concreta de humanos que representen un otro pero que a su vez esta otredad refleje cierta similitud con las propias conductas tanto lingüísticas y discursivas como materiales y sociales. ${ }^{17}$

Así, el cuerpo de los insectos no presenta la materialidad de un cuerpo en sí, sino, tal como se establece en la idea de devenir-animal de Deleuze y Guattari, el conjunto de elementos que lo establecen dentro de sí y para con su entorno. Sería indistinto, entonces, si los insectos son corporalmente humanos o se presentan como humanizados ya que sus acciones y habla compositiva son parte de una multiplicidad constitutiva que tiende hacia lo animal. Entonces, ¿cómo se resignifican los nombres y códigos humanos ante el uso por parte de los insectos? Como se mencionó en relación al cuerpo, el devenir no implica una imitación ni una corporalidad concreta, sino que compete

a partir de las formas que se tiene, del sujeto que se es, de los órganos que se posee o de las funciones que se desempeña, extraer partículas, entre las que se instauran relaciones de movimiento y de reposo, [...] las más próximas a lo que se está deviniendo, ygracias a las cuales se deviene (Deleuzey Guattari, 2015, p. 275).

Es entonces que el devenir insecto de los personajes solo puede suceder si se llevan a cabo elementos, relaciones de movimiento, medios, de partículas de insectos. Sin perder de vista que, si los devenires corresponden a colectividades menores, su relación con el uso y el espacio irlandés no resulta aleatoria, sino más bien necesaria e imperante.

\section{Las variantes lingüísticas y comunicativas de Rhapsody in Stephen's Green}

En relación a Cruiskeen Lawn, Tracy sugiere que "the central concern was always language, its use and misuse" (1994, p. 4). Lo mismo podría decirse de Rhapsody in Stephen's Green puesto que el texto dramático se centra en las interacciones que tienen los insectos en escena. Sin olvidar, a su vez, que una de las mayores preocupaciones de la obra reside en una emulación cuidadosa de distintas formas de habla identificadas como pertenecientes a distintas partes de Irlanda. De hecho, podría decirse que el argumento de la trama es escaso y, así como en el caso de Faustus Kelly, "all words and no play makes Faustus Kelly a dull boy" (Jernigan, 2013, viii). Es entonces que la palabra se puede pensar como uno de los elementos centrales de Rhapsody in Stephen's Green.

Si el espacio del parque por la noche conforma un no-espacio por fuera de la esfera humana, es decir un espacio animal, este también se compone por fuera

17 En el caso de la primera representación, Hilton Edwards menciona que: "There are two ways of approaching these insects theatrically; one, to make the humans as insect-like as possible by covering up; or, two, to adapt the human figure; i.e. Mr Beetle in a shiny American cloth morning coat with tails to the ground, and bowler hat" (Long, 2018, p. 126). 
de la norma lingüística instituida y aceptada, esto es, dentro de un uso menor de la lengua. Las variedades lingüísticas que competen a los insectos mencionados anteriormente componen un habla que tiene una referencia tanto compartida por el lenguaje humano y constituido por él, pero sobre todo por los lectores y posibles espectadores. De esta manera, y de forma similar a como Kager analiza el tipo de lenguaje presentado en Cruiskeen Lawn, "the smallest differences in pronunciation are rendered with care" (2017, p. 68). La transferencia de esta capacidad de decir y, consecuentemente, de pertenecer por parte de los insectos se torna un tanto cómica por la cantidad desmedida de recursos que utiliza tanto en la descripción de la apariencia humanizada de los insectos como en la emulación de distintos tipos de acentos locales.

Por un lado, el texto dramático cuenta con didascálicas que direccionan esta emulación de la oralidad y que especifican la forma en la que debería hablar cada personaje "In an appalling Dublin accent, apparently even flatter tan the Tramp's” (O’Brien, 2013, p. 186); “The duck's voice is sinister and high-pitched and it speaks with a most refined foreign accent” (ibíd., p. 192); "Enter Mr. And Mrs. Cricket. Both speak with the rawest of all possible Cork accents" (ibíd., p. 196). Por otro lado, se registra una emulación de la oralidad en la grafía que olvida ciertas convenciones escriturarias del inglés en pos de lograr una reproducción fonética de cómo debería sonar aquello que se lee -“An' isn't it worth puttin' yourself into a lather for-a pile of stuff that cost us the grey hairs of a lifetime to put together?" (ibíd., p. 186); “No, chickabiddy, mustn't come out, juste at what daddy gave you now” (ibíd., p. 192); "Mind oorself now” (ibíd., p.196). Así, se ven presentadas distintas formas de habla, encarnadas en distintos grupos de insectos, pertenecientes a diferentes partes del país, estableciendo una tensión "between urban and rural accents, and between the perceived neutrality of 'educated' pronunciation against the stronger inflections of the working classes" (Long, 2017, pp. 46-47).

Específicamente, las abejas del acto I presentan diferentes variantes dentro del propio inglés dublinés. Entre algunas de estas, se destacan la pronunciación de la $i$ como ee - "mean eet"- y la o como ao -"aold boy"-. Con el acto II, y como se mencionó anteriormente, la representación de la oralidad se expande al presentar a nuevos insectos aparejados de nuevos acentos, también, vinculados a la espacialidad irlandesa: los escarabajos, aún con acento dublinés, presentan un cambio de $m y / b y$ por me/be -"seen meeself"- y el uso de la o lo cambian por una w -"bew-uks"'“wan June“-, el pato, presentado como foráneo, abrevia el do you por dýou y también presenta un cambio similar en el uso de la vocal o y los grillos, provenientes de Cork, cambian el you por una doble oy la wh por una ph -“do oo know phat happpened him. Could oo guess?"-. De este modo, este alejamiento del inglés estándar, antes que ser una experimentación lingüística, busca ser un retrato discursivo detallado, por momentos desmedido, de distintos espacios a partir del uso de distintos insectos. Si bien se escucharía una forma de hablar distintiva para cada clase de insecto que se concentra en el acento particular que se le asigna a cada uno ligado a la región o especialidad que habitan, las formas particulares de expresarse también están acompañadas de diferentes formas de actuar que varían entre los grupos de insectos. 
Lenguaje y comunicación de insectos en Rhapsody...

Entre los distintos insectos que se presentan a lo largo de las escenas, con sus formas de oralidad particulares y fuertemente ancladas en regionalismos, hay poca interacción. Las escenas se desarrollan, como se mencionó anteriormente, con cierta mediación del vagabundo. De esta manera, se establecen bloques de diálogo entre insectos del mismo tipo que luego se encadenan con conversaciones de insectos de otro tipo, generando un diálogo fluido entre insectos de la misma clase.

En la posibilidad de pensar a estos personajes como insectos humanizados, aquí surge una nueva apropiación animal de conductas humanas. Es decir, la dificultad de establecer un diálogo prolongado y comprensivo con una especie diferente a la propia. Solomon también resalta estos problemas de comunicación en la producción dramática del autor, ya que las obras “work best when they interrogate this very assumption of spoken lines of dialogue as transparent expressions of characters' thoughts, and there are explicit reflections on the problems of the spoken word in each of them" (2014, p. 69).

Si bien los humanos establecen comunicación lingüística con otros humanos, tal como establece Benveniste en Problemas de lingüística general I, estos no pueden instaurar el dialogo por fuera de la esfera humana. Esto se replicaría en esta coexistencia de insectos donde, en los pocos diálogos entre especies, la comunicación se torna dificultosa. Antes de resultar problemática, esta imposibilidad se torna cómica al resaltar la emulación excesiva de la oralidad y el escaso entendimiento entre personajes.

Este tipo de interacción deficiente entre insectos se puede pensar a partir de la problematización que hace Myles del uso del lenguaje. Tanto en esta obra como en diversas entregas de Cruiskeen Lawn, el autor presenta su "mistrust of language as an instrument of knowledge and literal representation of the world" (Coulouma, 2015, p. 100). Si la aprehensión de un lenguaje requiere la comprensión dentro de un sistema de significados más amplios relacionado con lo social y lo cultural, aquí lo que sucede entre los insectos no es una comprensión total, sino parcial.

La idea de "sub-aprehensión" presenta un tipo de aprehensión donde el receptor no es totalmente consciente de las implicancias de lo que escucha. ${ }^{18}$ Así, se conforma como un tipo de comprensión incompleta que, como el prefijo sugiere, asigna formas leves de malentendidos que realzan la distancia entre discurso y realidad. Esto, en el caso de de Rhapsody in Stephen's Green, se ve reforzado por los usos de la oralidad de los insectos ya mencionados y la visión de mundo que viene aparejada con estos. A saber, el intercambio entre la señora grillo y la señora escarabajo:

Mrs. Beetle: Sure you can't have a proper home without a pile. Nor you can't have happiness nor a future. A pile is what keeps a home together, woman dear.

18 Este concepto es parte de una tríada, acompañado por "des-aprehensión" y "sobre-aprehensión", expuestos en Abella, (2020) que responde a la problematización de la aprehensión y uso del lenguaje en contextos multilingües y de diglosia que pueden leerse en algunas de las obras de O’Nolan. 
Mrs. Cricket: Ah yerra sure there's nothing like a grand nice little houseen for keepin' a home together, and a nice job in the service for the man with a grand pinshin at the end of it (O'Brien, 2013, p. 203).

Lo que sucede en este intercambio entre especies de insectos es que la comunicación no se da de manera fluida ya que cada insecto presenta una fijación en su propio discurso. El lenguaje aparece como un correlato, es decir, una visión de mundo. En el caso de los insectos de Rhapsody in Stephen's Green, la propia configuración lingüística comunica ciertos valores y miradas de mundo que son difíciles de comprender para los insectos receptores del discurso y, por esto mismo, es que la aprehensión es parcial.

En el ejemplo citado, tenemos a la señora escarabajo que durante todo el acto II está obsesionada con cuidar de su "pila" que, ante la primera interacción ya sea con otros insectos o con el vagabundo, lo único que sabe decirle es la importancia de tener una "pila". Aquí, en particular, se lo expresa a la señora grillo que antes de comprender lo que esta "pila" significa responde con su propia concepción de modelo familiar. Si bien la señora escarabajo presenta esta idea de acumular riqueza como base del hogar, la señora grillo repara en la calidad de la casa y del trabajo en vistas de una vida próspera. Es de esta manera que tanto en este fragmento como en otras interacciones de la obra cada especie impone un orden establecido no solo al hablar, es decir lingüísticamente, sino con sus acciones y el campo de conocimientos sociales y culturales que las rodean.

\section{Conclusión}

A modo de conclusión, bajo la firma de Myles na gCopaleen, Rhapsody in Stephen's Green establece al lenguaje como elemento central de la dramaturgia y la primera representación dramática en 1943 con toda la polémica que trajo luego de su estreno. Un lenguaje que, a su vez, elimina el límite establecido entre hombre y animal, dotando de capacidades sociales y comunicativas a los insectos que habitan Stephen's Green.

Se puede decir, entonces, que la obra en cuestión presenta distintos grupos de insectos cada uno conformado por características constitutivas que a su vez diagraman un retrato de los actores de la sociedad irlandesa a partir de su habla y de las conductas que le son aparejadas. En este sentido, y teniendo en cuenta el valor que se le adscribe a la posibilidad de igualar una clase de insecto a cierta zona del mapa irlandés, con el correr de la escenas se constituye un cierto valor identitario no solo de la sociedad en general, sino de cada una de estas clases de insectos en particular. La idea que reina de forma anterior y posterior a la conformación de la república que equivale a la nación y al lenguaje está presente. Este valor, de cualquier manera, al ser transferido a los insectos y por el tipo de interacciones que establecen, toma un tinte burlón; la pronunciación, las palabras y el discurso se vuelven exagerados dentro de cuerpos animales. De este modo, 
los insectos exceden la esfera animal para ser vehículos de correspondencias humanas que se tornan grotescas, sobreactuando la capacidad de pertenencia desde el lenguaje y desde el accionar a espacios que los espectadores reconocen.

En este sentido, la adaptación de la obra de los hermanos Čapek busca anclarse en una geografía local, cercana al posible espectador del Gaiety Theatre, con referencias al parque dublinés y su entorno, y a partir del habla de sus personajes. La equiparación de una cierta forma de habla a cierto insecto es la pauta con la que se conforman los personajes a través de los actos y sus interacciones. Así, la comunicación posible entre animales, planteada por Benveniste, es totalmente sobrepasada y se establece una comunicación lingüística propia de la esfera humana. No obstante, esta no es la única característica humana que se le atribuye a los insectos: las didascalias mencionan que los insectos tienen elementos humanos y los diálogos de interpelación entre insectos cuentan con categorías humanas.

No obstante, es menester reiterar que esta conformación de ciertos grupos sociales en clases de insectos está fuertemente ligada al uso de regionalismos lingüísticos que se detalló en secciones anteriores. Así, como asegura Vicent Descombes, "la incomodidad que produce entre nosotros la noción de identidad colectiva es antes que nada una incomodidad ligada al lenguaje” (2015, p. 182). El lenguaje establece una capacidad diferenciadora y porta consigo misma la capacidad de poner en un mismo plano a aquellos que la usan de la misma forma y de separarse de aquellos que hacen otro tipo de usos. Aquí, esa capacidad que podría pensarse como humana debido a su cualidad lingüística y dialógica, se vislumbra dentro de la esfera animal con un excesivo detalle ligada a lo irlandés y en el armado de los distintos regionalismos y sus formas de habla.

Asimismo, la figura del vagabundo es central para pensar la relación entre insectos y humanos durante toda la obra. Este personaje no solo establece contacto con los insectos sino que también funciona como mediador en la sucesión de intercambios entre ellos y expone su propio juicio sobre estas especies que observa y lo que considera que es la suya. El rechazo a la esfera animal ante actividades como matar y comer, así como la falta de organización, antes de generar un distanciamiento real con la esfera animal, solo relativiza el comportamiento humano que se ve, también, claramente envuelto sobre este tipo de lógicas de acción. De este modo, la separación entre el mundo del vagabundo y los sucesos que ocurren en el parque por la noche se relativiza, haciendo de los personajes figuras complejas insecto-humano que comparten elementos de ambos mundos y que no pueden delinear con claridad el alcance de una categoría sobre la otra. Más bien, hay un hacer cuerpo con el animal, un devenir-insecto por parte de los personajes.

Es así que Rhapsody in Stephen's Green pone en escena una representación de clichés culturales y ofrece un acercamiento a una posible problematización de la autenticidad de los actores sociales dentro de una nación a partir de sus usos de regionalismos exacerbados y, antes que conformar retratos verosímiles e íntimamente ligados a la realidad del posible espectador, compone una complejidad identitaria a partir de una transferencia animal que por momentos se torna desmedida y burlona. 


\section{O Bibliografía}

"Abella, J. (2020). Understanding and mis-understanding in language in Brian O’Nolan's An Béal Bocht and Cruiskeen Lawn. Estudios Irlandeses, 15, 1-12.

"Agamben, G. (2006). Lo abierto: el hombreyel animal. Buenos Aires: Adriana Hidalgo.

" Asensio Peral, G. (2018). 'One does not take sides in these neutral latitudes': Myles na gCopaleen and The Emergency. International Journal of English Studies, 18(1), 1-16.

" Aristóteles (2000). Parte de los animales. Marcha de los animales. Movimiento de los animales. Madrid: Gredos.

" Benveniste, E. (1997). Problemas de lingüística general I. México: Siglo XXI editores.

" Coulouma, F. (2015). Diglossia and the Linguistic Turn: Flann O'Brien's Philosophy of Language. Dublín: Dalkey Archive Press.

" Cragnolini, M.B. (2016). Extraños animales: filosofía y animalidad en el pensar contemporáneo. Buenos Aires: Prometeo Libros.

"Deleuze, G. y Guattari, F. (1990). Kafka: por una literatura menor. México D. F.: Ediciones Era.

"Deleuze, G. y Guattari, F. (2015). Mil mesetas (capitalismo y esquizofrenia). Valencia: Pre-textos.

"Derrida, J. (2008). El animal que luego estoy si(gui)endo. Madrid: Trotta.

"Descombes, V. (2015). El idioma de la identidad. Buenos Aires: Eterna Cadencia.

" Girvin, B. (2006). The Emergency: Neutral Ireland, 1939-45. London: Macmillan.

" Hachey, T. E. (2002). The Rhetoric and Reality of Irish Neutrality. New Hibernia Review, 6, 26-43.

" Heidegger, M. (2007), Los conceptos fundamentales de la metafísica. Mundo, finitud y soledad. Madrid: Alianza.

" Jernigan, D. (2013). Introduction. En O’Brien, F. Plays \& Teleplays (pp.vii-xx). Dublín: Dalkey Archive Press.

"Kager, M. (2017). Lamhd láftar and bad language: bilingual cognition in Cruiskeen Lawn. En Borg, R.; Fagan, P. y McCourt, J. (eds.). Flann O'Brien: Problems with Authority (pp. 54-70). Cork: Cork University Press.

" Long, M. (2014). An Béal Bocht, Translation and the Proper Name. En Murphet, J.; Mc Donald, R. y Morrell, S. (eds.). Flann O'Brien \& Modernism (pp. 102-118). Londres: Bloomsbury.

" Long, M. (2017). 'No more drunk, truculent, witty, celtic, dark, desperate, amorous paddies!': Brian O'Nolan and the Irish Stereotype. En Borg, R.; Fagan, P. y McCourt, J. (eds.). Flann O'Brien: Problems with Authority (pp. 34-53). Cork: Cork University Press.

" Long, M. (ed.). (2018). The Collected Letters of Flann O’Brien. Dublín: Dalkey Archive Press.

"Nietzsche, F. (1998). Así habló Zaratustra. Madrid: Alianza.

" O’Brien, F. (2013). Plays \& Teleplays. Dublín: Dalkey Archive Press.

Solomon, S. (2014). 'The Outward Accidents of Illusion': O'Brien and the Theatrical. 
Lenguaje y comunicación de insectos en Rhapsody...

En Murphet, J.; Mc Donald, R. y Morrell, S. (eds.). Flann O’Brien \& Modernism (pp. 6176). Londres: Bloomsbury.

"Taaffe, C. (2008). Ireland through the Looking Glass: Flann O'Brien, Myles na gCopaleen and Irish Cultural Debate. Cork: Cork University Press.

" Tracy, R. (1994). Introduction. En O'Brien, F. Rhapsody in St Stephen's Green: The Insect Play (pp. 1-17). Dublín: The Lilliput Press. 